\title{
Decisions on the European Spallation Source
}

To the Editor - Theodore Roosevelt in his dry and direct style of speaking once said, "In any moment of decision, the best thing you can do is the right thing, the next best thing is the wrong thing, and the worst thing you can do is nothing." These are striking words to keep in mind when it comes to decisions on scientific investments with enormous impact over several decades.

To comprehend the importance of bringing a large scientific infrastructure to life, one only needs to consider the benefits and discoveries that were brought about by other European scientific facilities. A striking example is of course the European particle research institution CERN, whose investigation into the understanding of matter and the origins of the Universe also led to the development of innovative technology such as the World Wide Web. A decision to establish another European scientific facility finally appears on the horizon, the long-awaited European Spallation Source (ESS), a next-generation, high-flux neutron source for Europe ${ }^{1}$. There are three candidate sites to host the ESS. What is needed now is a decision to build it.

The key advantage of the ESS for scientific research is that it uses a highpower accelerator to propel protons at a target to produce well-defined pulses of neutrons. The peak flux of the neutron pulse is much higher than that achieved in reactor-based sources, such as the Institut Laue-Langevin (ILL) in Grenoble and the Forschungsneutronenquelle Heinz Maier-Leibnitz (FRM II) in Munich. Indeed, reactor sources have reached their limit in terms of the maximum neutron flux that can be delivered for science, and spallation sources are the only viable opportunity available to produce higher neutron fluxes. The high neutron fluxes and the advanced neutron methods available at the ESS will allow scientists to look deep inside the structure of materials with unrivalled spatial and temporal resolution. The scientific knowledge gained from research at the ESS will not only help us to satisfy human scientific curiosity but will also contribute to the solution of complex problems ranging from efficient energy production and storage to medical advances, and will unlock the secrets of new materials and novel states of matter.
Europe can pride itself in having the most sophisticated and well-equipped neutron science community in the world, operating the world's most powerful research reactors (ILL and FRM II) and the world's most successful spallation source (ISIS in the UK), as well as several powerful and innovative national and regional facilities. However, the opportunities opened by megawatt spallation sources such as the proposed ESS have not failed to be noticed by the United States and Japan, and indeed these countries have now forged ahead of Europe. In Japan the new spallation source J-PARC is close to performing its first user experiments, while in the Oak Ridge National Laboratory, Tennessee, the Spallation Neutron Source (SNS) is operating and instrumenting its first target station and has began to construct a second.

\section{There are three candidate sites to host the ESS. What is needed now is a decision to build it.}

European plans for a spallation source started in Jülich, Germany, in the 1980s with the Spallations-Neutronenquelle and evolved into a sophisticated design for the ESS in the 1990s. This essentially provided the basis for both the SNS and J-PARC. Unfortunately, there is no clear path to decide on scientific infrastructure at a European level. The first attempt to build the ESS in Germany originated at the national level and never reached the point of a formal decision ${ }^{2}$. With the construction, commissioning and imminent success of the SNS and J-PARC, the pressure on Europe to capitalize on its own innovation in neutron science has mounted. Interest and support in the community has been enormous, impelling several countries to put forward candidate sites to host the ESS: Bilbao in Spain, Debrecen in Hungary, and Lund in Sweden. The need and the wide interest of the European scientific community in the ESS has been noticed; in a recent review by the European Strategy Forum on Research Infrastructures, the ESS was placed high on its list of priorities. However, the decision to locate and build the ESS goes beyond national boundaries and requires the support of several European countries.

What is needed now is a decision. A series of meetings is taking place in May that, under the chairmanship of the Czech Republic, will gather all European countries interested in contributing to the ESS. Because of the quirkiness of EU institutions it is a faux pas for a country to have a decision in its favour while it holds the rotating EU presidency. Unfortunately it seems that Murphy's law is working here. After the current presidency of the Czech Republic, the next countries to assume this post will be Sweden, Spain, then a break with a non-bidding Belgium, and then Hungary.

This means that if a decision is not taken before the end of the Czech term, there will be no decision on the ESS for a further two years, dealing a monumental blow to neutron science. One should not feel that the curtain will fall on European neutron science if there is no decision, but the allure of large scientific investments in the United States announced by the Obama administration $^{3}$ and the presence of a powerful spallation source there will be a siren call for Europe's best and brightest scientists. This will deprive Europe of talent and expertise and will seriously jeopardize its future capability in this area. We would hope that when European countries sit down to decide on the fate of the ESS they will recall the words of Theodore Roosevelt. There are three suitable sites that are willing and able to host the ESS. By choosing one of them they will be sending a positive message that neutron science in Europe will have a bright future.

Dimitri N. Argyriou and Heloisa Nunes Bordallo Helmholtz-Zentrum für Materialen und Energie Berlin, Glienicker Strasse 100, 14109 Berlin, Germany. e-mail: argyriou@helmholtz-berlin.de; bordallo@helmholtz-berlin.de. 